\title{
On the mean square formula of the error term in the Dirichlet divisor problem
}

\author{
Y.-K. Lau \& K.-M. Tsang \\ Department of Mathematics, The University of Hong Kong, Pokfulam Road, Hong Kong \\ e-mail: yklau@maths.hku.hk, kmtsang@maths.hku.hk
}

\section{INTRODUCTION}

Let $d(n)$ denote the divisor function. In 1849, Dirichlet introduced an elementary but important method to show a quite precise asymptotic formula for its summatory function,

$$
\sum_{n \leq t} d(n)=t(\log t+2 \gamma-1)+\Delta(t)
$$

with $\Delta(t)=O\left(t^{1 / 2}\right)$. Since then, there are plenty of works in the literature devoted to exploring this error term on various aspects, including for instance, its high moments, distribution function, gaps between sign-changes and omega results (see [8], [20], [5], [6], [18], etc).

In this paper we are concerned with the size of remainder term $F(x)$ in the mean square formula

$$
\int_{2}^{x} \Delta(t)^{2} d t=\frac{1}{6 \pi^{2}} \sum_{m=1}^{\infty} \frac{d(m)^{2}}{m^{3 / 2}} x^{3 / 2}+F(x) .
$$

The formula (1.2) with $F(x)=O\left(x^{5 / 4+\epsilon}\right)$ was first proved by Cramér[2] in 1922. It reveals $\Delta(t) \sim$ $t^{1 / 4}$ on average, and suggests the conjecture that $\Delta(t) \ll t^{1 / 4+\epsilon}(t \rightarrow \infty)$ holds for any positive $\epsilon$. This is a difficult conjecture and the best exponent of $t$ to date is $131 / 416(=1 / 4+27 / 416)$, due to Huxley [7]. The size of $F(x)$ governs the quality of the mean square of $\Delta(x)$ over short intervals

$$
\int_{x}^{x+L} \Delta(t)^{2} d t \sim c x^{1 / 2} L,
$$

which follows from $(1.2)$ once $F(x)=o\left(x^{1 / 2} L\right)$. Such results for short intervals show the local behaviour of $\Delta(t)$ (see [15] and [12] for relevant works).

Cramér's estimate on $F(x)$ is not sharp. In 1955, Tong[19] improved it to $F(x)=O\left(x \log ^{5} x\right)$, which then remained unchallenged for the next three decades, until it was subsequently refined to

$$
F(x)=O\left(x \log ^{4} x\right)
$$

by Preissmann[17]. Further progress towards its size may be hard because, as shown in [11], $F(x)=\Omega_{-}\left(x \log ^{2} x\right)$. In other words, the room for further improvement is not more $\operatorname{than} \log ^{2} x$. It is anticipated that the omega result is closer to the true magnitude, in virtue of the almost all result in [21]. Very recently, though unable to improve the bound for $F(x)$, Nowak[16] pushed forth the record in a closely analogous situation - the circle problem.

Let $P(t)$ denote the error term in the circle problem. Owing to the similarity between their Voronoi series, the methodology (based on these series) to handle $\Delta(t)$ usually applies to $P(t)$ and vice versa. Let

$$
Q(x):=\int_{2}^{x} P(t)^{2} d t-\frac{1}{3 \pi^{2}} \sum_{n=1}^{\infty} \frac{r(n)^{2}}{n^{3 / 2}} x^{3 / 2}
$$

where $r(n)$ denotes the number of ways of writing $n$ as a sum of two squares. In [17], Preissmann showed that $Q(x)=O\left(x \log ^{2} x\right)$ along the same line of argument in (1.3). On the other side we 
know $Q(x)=\Omega(x)$ by [10], so again the room for improvement on the bound of $Q(x)$ is at most $\log ^{2} x$. Remarkably, in [16] Nowak was able to shave off one-half from the log-power and got

$$
Q(x)=O\left(x(\log x)^{3 / 2} \log _{2} x\right) .
$$

Here and in the sequel, $\log _{r}$ denotes the $r$-th iterated $\log$ arithm, thus $\log _{2} x=\log \log x$. Like Preissmann[17], Nowak utilized the Hilbert-type inequality of Montgomery and Vaughan to bound $Q(x)$. But specializng to this case, he made an insightful use of the frequent vanishing of $r(n)$, and the wide spacing between integers that are sums of two squares. Thus he successfully gained the extra saving. Unfortunately the divisor function $d(n)$ does not possess this special vanishing property, and hence the argument in [16] does not work for $\Delta(t)$.

Our goal here is to propose a novel and flexible way of refinement by means of the correlated sum of the arithmetical function instead of the vanishing property. This will be carried out in two scenarios by the authors and the anonymous referee respectively. Both give improvements upon the estimate in (1.3) but indeed the referee's method is more efficient and yields a stronger result. As the ideas behind seem to be on different basis, we include both approaches and their resulting consequences (see Theorems 1 and 2 below). Theorem 1 is due to the authors, whose idea is to group terms with a similar value of $d(n)$ and then apply Hilbert's inequality to the products of these new subsums. Section 3 is devoted to its proof. The sharper result in Theorem 2 is accomplished in Section 4 with the ingenious proof of the anonymous referee. Our comprehension of the subtlety is to pinch the adjacent terms to make a more effective use of Hilbert's inequality and the trade-off is some error terms involving the correlated sum of the divisor function.

Theorem 1. Let $x \geq 2$ and define $F(x)$ as the remainder term in (1.2). Then, we have

$$
F(x)=O\left(x(\log x)^{7 / 2}\left(\log _{2} x\right)^{5 / 2}\right) .
$$

Theorem 2. The result in Theorem 1 can be improved to $F(x)=O\left(x(\log x)^{3}\left(\log _{2} x\right)\right)$.

These methods work for the error term $P(x)$, and plainly we deduce the following along the proof of Theorem 2 .

Theorem 3. For $x \geq 2$, we have $Q(x)=O\left(x(\log x)\left(\log _{2} x\right)\right)$ where $Q(x)$ is defined as in (1.4).

As is customary in this circle of research, the novel idea in the divisor problem can also be applied to the error function

$$
E(T):=\int_{0}^{T}\left|\zeta\left(\frac{1}{2}+i t\right)\right|^{2} d t-T \log \frac{T}{2 \pi}-(2 \gamma-1) T, \quad T>0 .
$$

Atkinson [1] gave an approximation to $E(T)$ in terms of two finite sums. The first sum is very similar to the Voronoi series for $\Delta(x)$, while the second sum is usually not difficult to handle. By using Atkinson's formula, Heath-Brown [3] proved that the remainder term

$$
F_{1}(T):=\int_{0}^{T} E(t)^{2} d t-c T^{3 / 2}, \quad\left(c=\frac{2}{3}(2 \pi)^{-1 / 2} \sum_{n=1}^{\infty} d(n)^{2} n^{-3 / 2}\right)
$$

satisfies $F_{1}(T) \ll T^{5 / 4} \log ^{2} T$. Later Meurman [13] derived a smoothened version of Atkinson's formula (see (5.1) below) in which the error term is much sharper, thereby got the better bound $O\left(T \log ^{5} T\right)$ for $F_{1}(T)$. (By a different approach, this same result was also obtained independently by Motohashi.) Then Preissmann (see [9, p.73]) applied the Montgomery-Vaughan inequality and further reduced this bound to $O\left(T \log ^{4} T\right)$. Now we can refine to the following.

Theorem 4. Let $T>0$ and let $F_{1}(T)$ be defined in (1.5). We have

$$
F_{1}(T)=O\left(T(\log T)^{3}\left(\log _{2} T\right)\right) .
$$

An outline of the proof is given in Section 5 . 


\section{Preparations}

To prove Theorem 1, we need the following variant of Hilbert's inequality.

Lemma 2.1. Let $\mathcal{A}$ and $\mathcal{B}$ be two sets of (distinct) real numbers such that either $\mathcal{A}=\mathcal{B}$ or $\mathcal{A} \cap \mathcal{B}=\emptyset$. Define

$$
\delta_{\mathcal{A} \cup \mathcal{B}}(\lambda):=\min _{\substack{\nu \in \mathcal{A} \cup \mathcal{B} \\ \nu \neq \lambda}}|\lambda-\nu|
$$

Given any two sequences $\left\{a_{\lambda}\right\}_{\lambda \in \mathcal{A}}$ and $\left\{b_{\mu}\right\}_{\mu \in \mathcal{B}}$ of complex numbers, we have, for all $T \geq 1$,

$$
\int_{0}^{T} \sum_{\substack{\lambda \in \mathcal{A}, \mu \in \mathcal{B} \\ \lambda \neq \mu}} a_{\lambda} \overline{b_{\mu}} e(2(\lambda-\mu) u) d u \ll\left(\sum_{\lambda \in \mathcal{A}} \frac{\left|a_{\lambda}\right|^{2}}{\delta_{\mathcal{A} \cup \mathcal{B}}(\lambda)}\right)^{1 / 2}\left(\sum_{\mu \in \mathcal{B}} \frac{\left|b_{\mu}\right|^{2}}{\delta_{\mathcal{A} \cup \mathcal{B}}(\mu)}\right)^{1 / 2} .
$$

Proof. For $\mathcal{A}=\mathcal{B}$, this is merely a reformulation of Lemma B in [16], whose proof is based on Hilbert's inequality in [14, Theorem 2]. When $\mathcal{A}$ and $\mathcal{B}$ are two disjoint sets, the same argument reduces to showing

$$
\left|\sum_{\substack{\lambda \in \mathcal{A}, \mu \in \mathcal{B} \\ \lambda \neq \mu}} \frac{a_{\lambda} \overline{b_{\mu}}}{\lambda-\mu}\right| \ll\left(\sum_{\lambda \in \mathcal{A}} \frac{\left|a_{\lambda}\right|^{2}}{\delta_{\mathcal{A} \cup \mathcal{B}}(\lambda)}\right)^{1 / 2}\left(\sum_{\mu \in \mathcal{B}} \frac{\left|b_{\mu}\right|^{2}}{\delta_{\mathcal{A} \cup \mathcal{B}}(\mu)}\right)^{1 / 2} .
$$

To this end, we consider the two sequences $\left\{\widehat{a}_{\nu}\right\}_{\nu \in \mathcal{A} \cup \mathcal{B}}$ and $\left\{\widehat{b}_{\nu}\right\}_{\nu \in \mathcal{A} \cup \mathcal{B} \text { defined as }}$

$$
\widehat{a}_{\nu}=\left\{\begin{array}{ll}
a_{\lambda} & \text { if } \nu=\lambda \in \mathcal{A}, \\
0 & \text { otherwise, }
\end{array} \quad \text { and } \quad \widehat{b}_{\nu}= \begin{cases}b_{\mu} & \text { if } \nu=\mu \in \mathcal{B} \\
0 & \text { otherwise }\end{cases}\right.
$$

Then (2.1) is plainly a consequence of Hilbert's inequality applied to $\left\{\widehat{a}_{\nu}\right\}$ and $\left\{\widehat{b}_{\nu}\right\}$.

Lemma 2.2. Let $y \geq 2$ and assume $1 \leq|h| \leq y^{5 / 6}$. Then

$$
\sum_{m \leq y} d(m) d(m+h) \ll \sigma_{-1}(|h|) y \log ^{2} y
$$

where $\sigma_{-1}(h)=\sum_{d \mid h} d^{-1}$.

Proof. The case of positive $h$ follows immediately from [4], or see [11, p.83]. Replacing $m+h$ by $m$ then yields the other case.

\section{Proof of Theorem 1}

Let us fix any sufficiently large number $x$, and define $F(x)$ as in (1.2). Then, by equation (2.2) in [11], we have

$$
F(x)=S(x)+S_{1}(x)+O(x)
$$

where

$$
S(x)=\left(4 \pi^{2}\right)^{-1} \sum_{m \neq n \leq x^{7}} \frac{d(m) d(n)}{(m n)^{3 / 4}} \int_{2}^{x} \sqrt{u} \cos (4 \pi(\sqrt{m}-\sqrt{n}) \sqrt{u}) d u
$$

and

$$
S_{1}(x)=\left(4 \pi^{2}\right)^{-1} \sum_{m, n \leq x^{7}} \frac{d(m) d(n)}{(m n)^{3 / 4}} \int_{2}^{x} \sqrt{u} \sin (4 \pi(\sqrt{m}+\sqrt{n}) \sqrt{u}) d u .
$$

(This is shown by using the good approximation to $\Delta(x)$ developed by Meurman[13] with some standard arguments.) 
We show that the double sum $S_{1}(x)$ is negligible in comparison with our result. Indeed, by integrating by parts, the integral is $\ll x(\sqrt{m}+\sqrt{n})^{-1}$. Hence we have

$$
S_{1}(x) \ll x \sum_{m \leq n \leq x^{7}} \frac{d(m)}{m^{3 / 4}} \frac{d(n)}{n^{5 / 4}} \ll x \log ^{3} x .
$$

Thus,

$$
F(x)=S(x)+O\left(x \log ^{3} x\right) .
$$

Now take $J:=\left[30 \log _{2} x\right]$ and for $0 \leq j \leq J$, define

$$
\mathcal{C}_{j}:=\left\{n \leq x^{7}: 2^{j-1}<d(n) \leq 2^{j}\right\}
$$

and $\mathcal{C}_{J+1}:=\left\{n \leq x^{7}: d(n)>2^{J}\right\}$. Then we can decompose $S(x)$ into

$$
S(x)=\left(4 \pi^{2}\right)^{-1} \sum_{i, j=0}^{J+1} S_{i j}(x)
$$

where

$$
S_{i j}(x)=\sum_{\substack{m \in \mathcal{C}_{i}, n \in \mathcal{C}_{j} \\ m \neq n}} \frac{d(m) d(n)}{(m n)^{3 / 4}} \int_{2}^{x} \sqrt{u} \cos (4 \pi(\sqrt{m}-\sqrt{n}) \sqrt{u}) d u .
$$

Applying a change of variable and the mean value theorem for definite integrals, we see that for some $\sqrt{2} \leq x_{1} \leq x_{2} \leq \sqrt{x}$

$$
\begin{aligned}
S_{i j}(x) & \ll x\left|\int_{x_{1}}^{x_{2}} \sum_{\substack{m \in \mathcal{C}_{i}, n \in \mathcal{C}_{j} \\
m \neq n}} \frac{d(m) d(n)}{(m n)^{3 / 4}} e(2(\sqrt{m}-\sqrt{n}) v) d v\right| \\
& \ll x\left(\sum_{m \in \mathcal{C}_{i}} \frac{d(m)^{2}}{m^{3 / 2} \delta_{i j}(m)} \sum_{n \in \mathcal{C}_{j}} \frac{d(n)^{2}}{n^{3 / 2} \delta_{i j}(n)}\right)^{1 / 2}
\end{aligned}
$$

by applying Lemma 2.1. Here we abbreviate $\delta_{i j}(m)$ for $\delta_{\mathcal{C}_{i} \cup \mathcal{C}_{j}}(m)=\min \underset{\substack{l \neq m \\ l \in \mathcal{C}_{i} \cup \mathcal{C}_{j}}}{ }|\sqrt{l}-\sqrt{m}|$.

Our main task is to bound the last product of two sums. The distance function $\delta_{i j}(m)$ has a quite trivial lower bound

$$
\delta_{i j}(m) \gg m^{-1 / 2}
$$

(which will also be explained below). Following from (3.6) and the well-known fact $\sum_{m \leq y} d(m)^{2} \ll$ $y \log ^{3} y$, one deduces that

$$
\sum_{m \in \mathcal{C}_{i}} \frac{d(m)^{2}}{m^{3 / 2} \delta_{i j}(m)} \ll \sum_{m \in x^{7}} \frac{d(m)^{2}}{m} \ll(\log x)^{4} .
$$

Inserting this into (3.5) and then (3.3), (3.2) yields the result of Preissmann[17] modulo a factor of $\left(\log _{2} x\right)^{2}$ (caused by the dyadic division in $(3.3)$ ).

Our novel idea refines the estimate for the sum in (3.7) when $i \leq j$. Let $0 \leq i \leq j \leq J+1$ and consider first the case $i \leq J$. We split

$$
\sum_{m \in \mathcal{C}_{i}} \frac{d(m)^{2}}{m^{3 / 2} \delta_{i j}(m)}
$$


into two parts according as $\delta_{i j}(m) \geq m^{-1 / 2} \log ^{2} x$ or not. Clearly, the subsum subject to $\delta_{i j}(m) \geq$ $m^{-1 / 2} \log ^{2} x$ is

$$
\ll(\log x)^{-2} \sum_{m \leq x^{7}} \frac{d(m)^{2}}{m} \ll \log ^{2} x .
$$

It remains to consider the subsum constrained by $\delta_{i j}(m)<m^{-1 / 2} \log ^{2} x$. For each $m$, there is an $l \in \mathcal{C}_{i} \cup \mathcal{C}_{j}$, not equal to $m$, such that $|\sqrt{m}-\sqrt{l}|=\delta_{i j}(m)$. The constraint on $\delta_{i j}(m)$ forces $m<\log ^{2} x$ if $l \geq 4 m$, and in this case, $\delta_{i j}(m) \geq \sqrt{l} / 2 \geq 1$. For $l<4 m$, we have $\delta_{i j}(m) \asymp|m-l| / \sqrt{m}$, which leads to (3.6) and $|m-l| \ll \log ^{2} x$ under the constraint. Thus we further divide this subsum into two pieces, running over $m<\log ^{2} x$ or otherwise. The piece over $m<\log ^{2} x$ is, by (3.6),

$$
\ll \sum_{m \leq \log ^{2} x} \frac{d(m)^{2}}{m} \ll\left(\log _{2} x\right)^{4} .
$$

Before estimating the other piece, we note that the value of $l$ depends on $m$. To get rid of the dependence, we take all possible $l$ with $|m-l| \ll \log ^{2} x$ into account. Letting $h=m-l$ (and thus $\left.\delta_{i j}(m) \gg|h| / \sqrt{m}\right)$, we bound this piece, up to a constant multiple, by

$$
\sum_{1 \leq|h| \ll \log ^{2} x}|h|^{-1} \sum_{\substack{m \geq \log ^{2} x \\(m, m+h) \in \mathcal{C}(i ; j)}} \frac{d(m)^{2}}{m}
$$

where $\mathcal{C}(i ; j):=\left\{(a, b): a \in \mathcal{C}_{i}, b \in \mathcal{C}_{i} \cup \mathcal{C}_{j}\right\}$. Then, under the assumption $i \leq j$ we observe that $d(m+h)>2^{i-1} \geq \frac{1}{2} d(m)$ for $m \in \mathcal{C}_{i}$ and $m+h \in \mathcal{C}_{i} \cup \mathcal{C}_{j}$. The inner sum in (3.8) can thus be replaced by the correlated sum, or more precisely, it is less than

$$
2 \sum_{m \leq x^{7}} \frac{d(m) d(m+h)}{m} \ll \sigma_{-1}(|h|) \log ^{3} x,
$$

by Lemma 2.2. As $\sum_{1 \leq|h| \ll \log ^{2} x} \sigma_{-1}(|h|) /|h| \ll \log _{2} x$, we infer that the double sum in (3.8) is $\ll \log ^{3} x \log _{2} x$, and consequently, for $0 \leq i \leq j \leq J+1, i \leq J$,

$$
\sum_{m \in \mathcal{C}_{i}} \frac{d(m)^{2}}{m^{3 / 2} \delta_{i j}(m)} \ll \log ^{3} x \log _{2} x .
$$

For the case $i=J+1$, a better upper bound follows, due to the sparsity of large values of the divisor function. Indeed, using (3.6) and $\sum_{m \leq y} d(m)^{3} \ll y \log ^{7} y$, we have

$$
\begin{aligned}
\sum_{m \in \mathcal{C}_{J+1}} \frac{d(m)^{2}}{m^{3 / 2} \delta_{J j}(m)} & \ll \sum_{m \in \mathcal{C}_{J+1}} \frac{d(m)^{2}}{m} \\
& \ll 2^{-J} \sum_{m \in \mathcal{C}_{J+1}} \frac{d(m)^{3}}{m} \\
& \ll 2^{-J} \sum_{m \in x^{7}} \frac{d(m)^{3}}{m} \\
& \ll 2^{-J} \log ^{8} x \ll 1
\end{aligned}
$$

by our choice of $J$, so (3.9) is also valid in this case.

In view of the condition $0 \leq i \leq j \leq J+1$, we are able to apply (3.9) to one of the two factors in the product in (3.5) (but not both unless $i=j$ ). The other factor is handled by (3.7). It follows that $S_{i j}(x) \ll x(\log x)^{7 / 2}\left(\log _{2} x\right)^{1 / 2}$. By (3.2) and (3.3), our proof of Theorem 1 is complete. 


\section{Proof of Theorem 2}

We give essentially verbatim the proof provided by the referee, which begins with the following robust lemma.

Lemma 4.1. Suppose $a_{n}$ are complex numbers for $N<n \leq 2 N$. Let $K \leq N$ be a positive integer. Then

$$
\sum_{N<m \neq n \leq 2 N} \frac{a_{m} \overline{a_{n}}}{\sqrt{m}-\sqrt{n}} \ll S_{1}+S_{2}+S_{3}
$$

where

$$
\begin{aligned}
& S_{1}=N^{3 / 2} K^{-1} \sum_{h \geq N / K} h^{-2} \sum_{N<n \leq 2 N-h}\left|a_{n} a_{n+h}\right|, \\
& S_{2}=N^{1 / 2} \sum_{h \leq N / K} h^{-1} \sum_{N<n \leq 2 N-h}\left|a_{n} a_{n+h}\right|
\end{aligned}
$$

and

$$
S_{3}=K N^{-1 / 2} \sum_{N<n \leq 2 N}\left|a_{n}\right|^{2} .
$$

Proof. To prove the lemma we divide $(N, 2 N]$ into $K$ disjoint intervals

$$
I=\left(\frac{k-1}{K} N, \frac{k}{K} N\right], \quad K<k \leq 2 K .
$$

We write $g(n)$ for the lower end-point of the interval $I$ for which $n \in I$, so that $g(n)<n \leq$ $g(n)+N / K$. We claim that

$$
\sum_{N<m \neq n \leq 2 N} \frac{a_{m} \overline{a_{n}}}{\sqrt{m}-\sqrt{n}}=\sum_{\substack{N<m, n \leq 2 N \\ g(m) \neq g(n)}} \frac{a_{m} \overline{a_{n}}}{\sqrt{g(m)}-\sqrt{g(n)}}+O\left(S_{1}\right)+O\left(S_{2}\right) .
$$

We first consider terms with $m \geq n+N / K$, so that $g(m) \neq g(n)$. Then $0<n-g(n) \leq N / K$, whence $0<\sqrt{n}-\sqrt{g(n)} \ll \sqrt{N} / K$, and similarly for $m$. We also have $\sqrt{m}-\sqrt{n} \gg(m-n) / \sqrt{N}$. Moreover if $m-n \geq 2 N / K$ then

$$
g(m)-g(n) \geq m-N / K-n \geq(m-n) / 2 .
$$

On the other hand, if $N / K \leq m-n<2 N / K$ we have

$$
g(m)-g(n) \geq N / K>(m-n) / 2,
$$

since $g(m) \neq g(n)$, as remarked above. In either case we conclude that

$$
\sqrt{g(m)}-\sqrt{g(n)} \gg(g(m)-g(n)) / \sqrt{N} \geq(m-n) / \sqrt{N} .
$$

It follows that

$$
\frac{1}{\sqrt{m}-\sqrt{n}}=\frac{1}{\sqrt{g(m)}-\sqrt{g(n)}}+O\left(\frac{N^{3 / 2}}{K(m-n)^{2}}\right)
$$

when $m \geq n+N / K$. Thus these $O$-terms contribute $O\left(S_{1}\right)$ to (4.1).

Secondly we consider terms in which $n<m<n+N / K$. Here we have $\sqrt{m}-\sqrt{n} \gg(m-n) / \sqrt{N}$. Moreover if $g(m)>g(n)$ we have $g(m)-g(n) \geq N / K$, whence $\sqrt{g(m)}-\sqrt{g(n)} \gg \sqrt{N} / K \gg$ $(m-n) / \sqrt{N}$. It follows that the terms under consideration contribute $O\left(S_{2}\right)$ to (4.1), which establishes the claim. 
We now group terms $n$ from a particular interval $I$, all of which have the same lower end-point $g(n)$. Thus, letting $I_{1}, \cdots, I_{J}$ be the sequence of intervals $I$ we set

$$
A(j)=\sum_{n \in I_{j}} a_{n}
$$

and we write $g_{j}$ for the lower end-point of $I_{j}$. Thus

$$
\sum_{\substack{N<m, n \leq 2 N \\ g(m) \neq g(n)}} \frac{a_{m} \overline{a_{n}}}{\sqrt{g(m)}-\sqrt{g(n)}}=\sum_{j \neq k} \frac{A(j) \overline{A(k)}}{\sqrt{g_{j}}-\sqrt{g_{k}}} .
$$

Moreover for $j>k$ we have $\sqrt{g_{j}}-\sqrt{g_{k}} \gg\left(g_{j}-g_{k}\right) / \sqrt{N} \gg \sqrt{N} / K$. We can now apply Hilbert's inequality (which is $(2.1$ ) with $\mathcal{A}=\mathcal{B}$ ) to give a bound

$$
\ll K N^{-1 / 2} \sum_{j \leq J}|A(j)|^{2}
$$

When we expand $|A(j)|^{2}$ we get terms $a_{n} \overline{a_{n+h}}$ with $n, n+h \in I_{j}$. Thus $|h| \leq N / K$. When $h \neq 0$ we have $K N^{-1 / 2} \leq N^{1 / 2}|h|^{-1}$, so that the overall contribution is $O\left(S_{2}\right)$. Finally, terms with $h=0$ produce a contribution $O\left(S_{3}\right)$, which completes the proof of Lemma 4.1.

We proceed now to prove Theorem 2. By (3.2) and a treatment as in the line before (3.5), there exist $a_{n} \in \mathbb{C}$ for $n \leq x^{7}$ such that $\left|a_{n}\right| \leq d(n) n^{-3 / 4}$ and

$$
F(x) \ll x(\log x)^{3}+x\left|\sum_{m \neq n} \frac{a_{m} \overline{a_{n}}}{\sqrt{m}-\sqrt{n}}\right| .
$$

Let $N_{j}=2^{j / 2}$ and decompose the sum on the left into $O\left((\log x)^{2}\right)$ subsums

$$
S_{i, j}=\sum_{\substack{N_{i}<m \leq \sqrt{2} N_{i} \\ N_{j}<n \leq \sqrt{2} N_{j}}} \frac{a_{m} \overline{a_{n}}}{\sqrt{m}-\sqrt{n}}
$$

where it is understood that $m \neq n$ if $i=j$. When $i \geq j+2$ we have $\sqrt{m}-\sqrt{n} \gg \sqrt{N_{i}}$, and a trivial bound yields $S_{i, j} \ll N_{i-j}^{-1 / 4}(\log x)^{2}$. Thus the total contribution from all such sums $S_{i, j}$ is $O\left((\log x)^{3}\right)$. Sums with $j \geq i+2$ may be handled similarly. We also have

$$
S_{i, i+1}+S_{i+1, i}=-S_{i, i}-S_{i+1, i+1}+\sum_{N_{i}<m \neq n \leq 2 N_{i}} \frac{a_{m} \overline{a_{n}}}{\sqrt{m}-\sqrt{n}} .
$$

The sum on the right may be bounded by Lemma 4.1, as can $S_{i, i}$ and $S_{i+1, i+1}$. We take $K=$ $\left[N_{i} /\left(1+\log N_{i}\right)\right]$ and find (via Lemma 2.2) that

$$
\sum_{N<n \leq 2 N-h}\left|a_{n} a_{n+h}\right| \ll N^{-1 / 2}(\log x)^{2} \sigma_{-1}(h),
$$

whence $S_{1} \ll(\log x)^{2}, S_{2} \ll(\log x)^{2}\left(\log _{2} x\right)$ and $S_{3} \ll(\log x)^{2}$. It follows that

$$
S_{i, i} \ll(\log x)^{2}\left(\log _{2} x\right) \quad \text { and } \quad S_{i, i+1}+S_{i+1, i} \ll(\log x)^{2}\left(\log _{2} x\right) .
$$

Thus on summing over $i \ll \log x$ we finally conclude that

$$
F(x) \ll x(\log x)^{3}\left(\log _{2} x\right) .
$$




\section{Proof of Theorem 4}

To prove Theorem 4, we begin with the modified version of Atkinson's formula as given by Meurman in [13]. Using the same notations in Meurman's paper,

$$
E(T)=\sum_{1}^{*}(T)+\sum_{2}^{*}(T)+\pi+O\left(T^{-1 / 4} \log T\right)
$$

where

$$
\begin{aligned}
& \sum_{1}^{*}(T)=\left(\frac{2 T}{\pi}\right)^{1 / 4} \sum_{n \leq(a+U)^{2}} \eta(n)(-1)^{n} d(n) n^{-3 / 4} e(T, n) \cos f(T, n), \\
& \sum_{2}^{*}(T)=-2 \sum_{n \leq Z(0)} \xi(T, n) d(n) n^{-1 / 2}\left(\log \frac{T}{2 \pi n}\right)^{-1} \cos g(T, n) .
\end{aligned}
$$

Here $T^{1 / 4} \ll U \ll T^{1 / 4}, T^{1 / 2} \ll a \ll T^{1 / 2}$. The sums $\sum_{1}^{*}(T)$ and $\sum_{2}^{*}(T)$ are essentially the same as those in the original formula of Atkinson, except for the smoothing functions $\eta(n)$ and $\xi(T, n)$, which approximate the characteristic functions on the intervals $\left[0,(a+U)^{2}\right]$ and $[0, Z(0)]$ respectively. With the functions $\eta(n)$ and $\xi(T, n)$, the $O$-term in (5.1) is much sharper. As was shown in [13, p.340-341]

$$
\int_{T}^{2 T} E^{2}(t) d t=I_{11}+2 I_{12}+I_{22}+O\left(T \log T+T^{1 / 2} I_{22}^{1 / 2}\right)
$$

where for $j, k \in\{1,2\}$,

Moreover,

$$
I_{j k}=\int_{T}^{2 T} \sum_{j}^{*}(t) \sum_{k}^{*}(t) d t .
$$

$$
I_{11}=c\left((2 T)^{3 / 2}-T^{3 / 2}\right)+M(T), \quad I_{22} \ll T \log ^{4} T
$$

with $M(T) \ll T \log ^{5} T$. This bound on $M(T)$ yields Meurman's result. In fact, apart from the harmless factor $(-1)^{n} \eta(n), M(T)$ is essentially the same as $F(x)$ in (3.1). Hence the idea in Section 4 proves

$$
M(T) \ll T(\log T)^{3}\left(\log _{2} T\right) .
$$

To complete the proof of Theorem 4, we establish the bounds

$$
I_{22} \ll T \log ^{3} T
$$

and

$$
I_{12} \ll T(\log T)^{3}\left(\log _{2} T\right)^{1 / 2}
$$

which are more than enough.

The proof of (5.4) follows the standard argument of opening up the square of $\sum_{2}^{*}(t)$ and then integrating term by term. By the compound angle formula, there is a subsum involving $\cos (g(t, n)+$ $g(t, m))$ which, by the mean value theorem, is

$$
\begin{aligned}
& \ll \sum_{n, m \leq Z(0)} \frac{d(n) d(m)}{\sqrt{n m}} \max _{T<t \leq 2 T}(\xi(t, n) \xi(t, m))\left(\log \frac{T}{2 \pi n} \log \frac{T}{2 \pi m}\right)^{-1} \\
& \ll\left(\sum_{n \leq Z(0)} d(n) n^{-1 / 2}\right)^{2} \ll T \log ^{2} T .
\end{aligned}
$$


Note that $Z(0) \leq(1-\delta) \frac{T}{2 \pi}$ for some $\delta>0$, depending on $a$. The other subsum, involving $\cos (g(t, n)-g(t, m))$, is

$$
\ll T \sum_{n \leq Z(0)} \frac{d(n)^{2}}{n}\left(\log \frac{T}{2 \pi n}\right)^{-2}+\sum_{m<n \leq Z(0)} \frac{d(n) d(m)}{\sqrt{n m}}\left(\log \frac{T}{2 \pi n} \log \frac{T}{2 \pi m} \log \frac{n}{m}\right)^{-1},
$$

again by applying the mean value theorem. Then partial summation together with the estimate $\sum_{m \leq y} d(n)^{2} \ll y \log ^{3} y$ shows the first sum here is $\ll \log ^{3} T$.

The second sum can be further splitted into two pieces. The one in which $m<n / 2, n \leq Z(0)$ is

$$
\ll \sum_{n \leq Z(0)} \frac{d(n)}{\sqrt{n}} \sum_{m \leq n} \frac{d(m)}{\sqrt{m}} \ll T \log ^{2} T .
$$

In view of the inequality $(\log (n / m))^{-1} \ll m /(n-m)$, the other piece is

$$
\begin{aligned}
& \ll \sum_{\frac{n}{2} \leq m<n \leq Z(0)} \frac{d(n) d(m)}{n}\left(\log \frac{T}{2 \pi n}\right)^{-2} \frac{m}{n-m} \\
& \ll \sum_{\frac{n}{2} \leq m<n \leq Z(0)} \frac{d(n) d(m)}{n-m} \\
& \leq \sum_{n \leq Z(0)} \sum_{m \leq n-n^{5 / 6}} \frac{d(n) d(m)}{n-m}+\sum_{n \leq Z(0)} \sum_{n-n^{5 / 6}<m<n} \frac{d(n) d(m)}{n-m} \\
& =\sum_{a}+\sum_{b},
\end{aligned}
$$

say. The $\operatorname{sum} \sum_{b}$ is

$$
\ll \sum_{h \leq Z(0)^{5 / 6}} h^{-1} \sum_{m \leq Z(0)} d(m) d(m+h) \ll T \log ^{3} T,
$$

by using Lemma 2.2. To bound the sum $\sum_{a}$, we first estimate the inner sum over $m$ by partial summation together with the formula (1.1), and it is $\ll \log ^{2} n$. Hence

$$
\sum_{a} \ll \sum_{n \leq Z(0)} d(n) \log ^{2} n \ll T \log ^{3} T .
$$

These together with (5.6) proves (5.4)

The proof of (5.5) follows the same argument of [13, p.341], where $\sum_{1}^{*}$ is splitted into two parts, according as $n \leq T / A$ and $T / A<n \leq(a+U)^{2}$ respectively. The contribution from the first part is $\ll T \log ^{2} T$, as shown in [13]. The contribution of the second part, by using Cauchy-Schwarz's inequality together with (5.4) and the bound for $M(T)$ in (5.3), is

$$
\ll\left\{\left(T \log ^{3} T+T \log ^{3} T \log _{2} T\right)\left(T \log ^{3} T\right)\right\}^{1 / 2} \ll T \log ^{3} T \log _{2}^{1 / 2} T .
$$

This completes the proofs of (5.4) and (5.5), and hence Theorem 3.

\section{Acknowledgement}

The authors wish to acknowledge the generous referee for providing the result in Theorem 2 and its proof.

The work described in this paper was fully supported by a grant from the Research Grants Council of the Hong Kong Special Administrative Region, China (HKU 7042/04P). 


\section{REFERENCES}

[1] F. V. Atkinson, The mean value of the Riemann-zeta function. Acta Math. 81 (1949), 353-376.

[2] H. Cramér, Über zwei Sätze von Herrn G. H. Hardy. Math. Zeit. 15 (1922), 200-210.

[3] D.R. Heath-Brown, The mean value theorem for the Riemann zeta function. Mathematika 25 (1978),177-184.

[4] D.R. Heath-Brown, The fourth power moment of the Riemann zeta function. Proc. London Math. Soc. 38 (1979), $385-422$.

[5] D.R. Heath-Brown, The distribution and moments of the error term in the Dirichlet divisor problem. Acta Arith. 60 (1992), 389-415.

[6] D.R. Heath-Brown and K. Tsang, Sign changes of $E(T), \Delta(x)$, and $P(x)$. J. Number Theory 49 (1994), 73-83.

[7] M.N. Huxley, Exponential sums and lattice points III. Proc. London Math. Soc. 87 (2003), 591-609.

[8] A. Ivić, Large values of the error term in the divisor problem. Invent. Math. 71 (1983), 513-520.

[9] A. Ivić, Mean values of the Riemann zeta function, Lecture on Maths. and Physics No.82, Tata Inst. Fund. Research, Bombay (1991), Springer, Heidelberg.

[10] A. Ivić, The Laplace transform of the square in the circle and divisor problems. Studia Sci. Math. Hungar. 32 (1996), 181-205.

[11] Y.-K. Lau and K.-M. Tsang, Mean square of the remainder term in the Dirichlet divisor problem. J. Théor. Nombres Bordeaux 7 (1995), 75-92.

[12] Y.-K. Lau and K.-M. Tsang, Moments over short intervals. Arch. Math. (Basel) 84 (2005), 249-257.

[13] T. Meurman, On the mean square of the Riemann zeta-function. Quart. J. Math. Oxford Ser. (2) 38 (1987), 337-343.

[14] H.L. Montgomery and R.C. Vaughan, Hilbert's inequality. J. London Math. Soc. 8 (1974), 73-82.

[15] W.G. Nowak, On the divisor problem: moments of $\Delta(x)$ over short intervals. Acta Arith. 109 (2003), 329-341.

[16] W.G. Nowak, Lattice points in a circle: an improved mean-square asymptotics. Acta Arith. 113 (2004), 259-272.

[17] E. Preissmann, Sur la moyenne quadratique du terme de reste du problme du cercle. C. R. Acad. Sci. Paris Sr. I Math. 306 (1988), 151-154.

[18] K. Soundararajan, Omega results for the divisor and circle problems. Int. Math. Res. Not. 2003, 1987-1998.

[19] K.-C. Tong, On divisor problems III. (Chinese) Acta Math. Sinica 6 (1956), 515-541.

[20] K.-M. Tsang, Higher-power moments of $\Delta(x), E(t)$ and $P(x)$. Proc. London Math. Soc. 65 (1992), 65-84.

[21] K.-M. Tsang, Mean square of the remainder term in the Dirichlet divisor problem II. Acta Arith. 71 (1995), 279-299. 